\title{
Redução de incertezas em radioterapia utilizando simulação Monte Carlo: análise espectral aplicada à correção de dose absorvida*
}

\author{
Reduction of uncertainties in radiotherapy assessed by Monte Carlo simulation: \\ spectral analysis applied to absorbed dose correction
}

Tatiana Marques ${ }^{1}$, Mirko Alva-Sánchez ${ }^{1}$, Patrícia Nicolucci $^{2}$

Resumo OBJETIVO: Determinar, por simulação Monte Carlo, os espectros de feixes de cobaltoterapia em profundidade na água e fatores de correção para doses absorvidas em dosímetros termoluminescentes de fluoreto de lítio. MATERIAIS E MÉTODOS: As simulações dos espectros secundários da fonte clínica de cobalto-60 foram realizadas com o código Monte Carlo PENELOPE, em diversas profundidades na água. Medidas experimentais de dose profunda foram obtidas com dosímetros termoluminescentes e câmara de ionização em condições de referência em radioterapia. Os fatores de correção para os dosímetros termoluminescentes foram obtidos através da razão entre as absorções relativas ao espectro de baixa energia e ao espectro total. RESULTADOS: A análise espectral em profundidade revelou a existência de espectros secundários de baixa energia responsáveis por uma parcela significativa da deposição de dose. Foram observadas discrepâncias de 3,2\% nas doses medidas experimentalmente com a câmara de ionização e com os dosímetros termoluminescentes. $O$ uso dos fatores de correção nessas medidas permitiu diminuir a discrepância entre as doses absorvidas para, no máximo, 0,3\%. CONCLUSÃO: Os espectros simulados permitem o cálculo de fatores de correção para as leituras de dosímetros termoluminescentes utilizados em medidas de dose profunda, contribuindo para a redução das incertezas associadas ao controle de qualidade de feixes clínicos em radioterapia. Unitermos: Simulação Monte Carlo; Radioterapia; Controle de qualidade; Espectrometria; Dosimetria termoluminescente; TLD.

Abstract OBJECTIVE: To calculate spectra of cobalt-60 beam at water depth and correction factors for absorbed dose measurements obtained with lithium fluoride thermoluminescent dosimeters using Monte Carlo simulation. MATERIALS AND METHODS: The simulations of secondary spectra of clinical cobalt-60 sources were performed with the PENELOPE Monte Carlo code at different water depths. Experimental measurements of deep doses were obtained with thermoluminescent dosimeters and ionization chamber under reference conditions for radiotherapy. Correction factors for the thermoluminescent dosimeters detectors were obtained through the ratio between the relative energy absorption for the low energy spectrum and the total spectrum. RESULTS: Deep spectral analysis has demonstrated the presence of secondary low-energy spectra responsible for a significant portion of the dose deposition. Discrepancies of $3.2 \%$ were observed among the doses measured with ionization chamber and thermoluminescent dosimeters. The adoption of correction factors has allowed a reduction in the discrepancy among absorbed doses to a maximum of $0.3 \%$. CONCLUSION: Simulated spectra allow the calculation of correction factors for reading of thermoluminescent dosimeters utilized in the measurement of deep doses, contributing for the reduction of uncertainties associated with quality control of clinical beams in radiotherapy.

Keywords: Monte Carlo simulation; Radiotherapy; Spectrometry; Quality control; Thermoluminescent dosimetry; TLD.

Marques T, Alva-Sánchez M, Nicolucci P. Redução de incertezas em radioterapia utilizando simulação Monte Carlo: análise espectral aplicada à correção de dose absorvida. Radiol Bras. 2010;43(2):119-123.

* Trabalho realizado no Hospital das Clínicas da Faculdade de Medicina de Ribeirão Preto da Universidade de São Paulo (HCFMRP-USP), Ribeirão Preto, SP, Brasil. Suporte financeiro: Conselho Nacional de Desenvolvimento Científico e Tecnológico (CNPq) e Coordenação de Aperfeiçoamento de Pessoal de Nível Superior (Capes).

1. Doutorandos, Pesquisadores do Departamento de Física Matemática da Universidade de São Paulo (DFM-USP), Ribeirão Preto, SP, Brasil.

2. Doutora, Professora do Departamento de Física e Matemática da Universidade de São Paulo (DFM-USP), Ribeirão Preto, SP, Brasil.

\section{INTRODUÇÃO}

A garantia da qualidade em tratamentos radioterápicos deve aliar alta precisão nos

Endereço para correspondência: Tatiana Marques. Universidade de São Paulo. Avenida Bandeirantes, 3900. Ribeirão Preto, SP, Brasil, 14040-901. E-mail: tatiana.marques@usp.br

Recebido para publicação em 26/3/2009. Aceito, após revisão, em 15/10/2009. planejamentos e nas doses prescritas com reprodutibilidade da técnica planejada, documentação minuciosa e cuidadosa dosimetria da situação planejada e do tratamento ${ }^{(1-3)}$. Características como homogeneidade e exatidão na dose absorvida são absolutamente relevantes para o sucesso de tratamentos clínicos em unidades de cobaltoterapia, especialmente em irradiações do 
corpo inteiro, que normalmente são combinadas com quimioterapia intensiva e transplante de medula óssea. Pequenos erros na determinação das doses em profundidade podem comprometer órgãos vitais, por exemplo, o pulmão ${ }^{(\mathbf{4 , 5})}$

A determinação das incertezas associadas aos tratamentos radioterápicos evidencia-se totalmente relevante para a garantia da eficiência de tratamentos clínicos. Uma fração considerável desta incerteza reside em características intrínsecas dos métodos e do aparato instrumental utilizado na determinação dos parâmetros dosimétricos, os quais são diretamente aplicados nos cálculos de prescrição de dose absorvida. Em busca da redução das incertezas associadas aos parâmetros dosimétricos, é viável utilizar a simulação Monte Carlo de interação da radiação ionizante com a matéria para identificar, quantificar e corrigir imprecisões advindas dos dosímetros utilizados no controle da qualidade em radioterapia.

Entre os dosímetros mais utilizados em radioterapia encontram-se os dosímetros termoluminescentes (TLDs), com características de número atômico efetivo $\left(\mathrm{Z}_{\text {efetivo }}\right.$ $=8,2$ ) próximo ao da água, alta resolução espacial de detecção (0,9 $\mathrm{mm})$ e baixo custo associado ao seu uso ${ }^{(6-8)}$. Os TLDs de fluoreto de lítio $(\mathrm{LiF})$ apresentam dependência energética para fótons com energia menor que $662 \mathrm{keV}^{(9)}$, o que implica a necessidade de correções em leituras realizadas com esses dosímetros quando expostos à radiação de baixas energias. Em medidas de dose absorvida em profundidade ${ }^{(\mathbf{1 0})}$, por exemplo, aplicadas ao controle de qualidade de irradiações do corpo inteiro ${ }^{(\mathbf{8})}$, o espectro de feixes de terapia em profundidades de interesse clínico é composto por parcelas de alta energia, para as quais o LiF responde de forma linear, e também por componentes de baixa energia, em que há dependência energética ${ }^{(9)}$.

A simulação de tratamentos clínicos baseada em método Monte Carlo tem sido largamente utilizada em radioterapia, devido principalmente à exatidão associada ao seu uso $^{(\mathbf{1 1})}$. O código de simulação Monte Carlo PENELOPE se apresenta como uma ferramenta bem estabelecida e eficiente para estudos de espectrometria e dosimetria de feixes clínicos, permitindo a representação geométrica fiel de diversos tipos de apare- lhos radioterápicos ${ }^{(\mathbf{1 2})}$. O código PENELOPE utiliza bibliotecas de secção de choque de materiais baseadas em normas internacionais $^{(\mathbf{1 3})}$, garantindo assim uma representação fidedigna das características de interação dos materiais de interesse dosimétrico.

Neste trabalho são apresentados os espectros de energia de um feixe clínico de cobalto-60 (Co-60) simulados em profundidade na água com o código de simulação Monte Carlo PENELOPE. Mediante análise quantitativa das contribuições em dose das componentes primária e secundária deste feixe, é possível calcular fatores de correção para as leituras de TLDs de LiF100 em diversas profundidades na água. A contribuição do uso dos fatores de correção em medidas de dose absorvida em TLDs pode ser realizada pela comparação entre as curvas de porcentagem de dose profunda (PDP), construídas com dados experimentais coletados com uma câmara de ionização e com TLDs, cujas respostas foram corrigidas por esses fatores.

\section{MATERIAIS E MÉTODOS}

\section{Simulação Monte Carlo}

As simulações computacionais de interação da radiação ionizante com a matéria foram realizadas com o pacote de simulação Monte Carlo PENELOPE, o qual engloba o código de transporte de partículas PENELOPE e o código de simulação de geometrias quadráticas PENGEOM. Nestas simulações, a fonte foi posicionada a $80 \mathrm{~cm}$ do objeto simulador, com uma abertura de 3,57 graus, que proporcionou um campo de radiação de $10 \times 10 \mathrm{~cm}^{2}$ na superfície do objeto simulador. $\mathrm{O}$ espectro primário de fótons é composto por dois picos, de mesma probabilidade de ocorrência, com energias de $1,17 \mathrm{MeV}$ e 1,33 $\mathrm{MeV}^{(\mathbf{1 2})}$, respectivamente. O objeto simulador foi um cubo de água, homogêneo, com $30 \mathrm{~cm}$ de aresta.

Os espectros de fótons em profundidade foram obtidos por meio do uso de detectores de impacto virtuais: pastilhas desenhadas virtualmente, simulando a geometria experimental dos TLDs, em profundidade dentro do objeto simulador, com 150 canais de energia distribuídos uniformemente entre $10 \mathrm{keV}$ e 1,33 MeV. Os detectores de impacto têm a função de contabilizar os fótons que alcançam a profundidade em que o detector foi posicionado, separando em canais e armazenando suas respectivas energias $^{(\mathbf{1 3})}$. Desta maneira, a resposta gerada ao final de cada simulação contém a função densidade de probabilidade associada à ocorrência de fótons, separadas por energia, em cada profundidade. A partir dos dados contidos nos arquivos de resposta, foram construídos os espectros totais e de baixa energia em diversas profundidades do objeto simulador. Foi também obtida uma curva de PDP simulada no eixo central do objeto simulador, para a determinação da acurácia do código PENELOPE neste tipo de simulação através da comparação com os dados experimentais de dose em profundidade.

\section{Dados experimentais}

As PDPs foram obtidas experimentalmente com uma câmara de ionização cilíndrica, tipo Farmer, de $0,6 \mathrm{~cm}^{3}$, e com TLDs de LiF-100, ambos posicionados em profundidade em um objeto simulador, cúbico, homogêneo de água, com aresta de $50 \mathrm{~cm}$, análogo ao simulado computacionalmente. O campo projetado na superfície do objeto simulador tinha $10 \times 10 \mathrm{~cm}^{2}$ e encontravase a uma distância fonte-superfície (DFS) de $80 \mathrm{~cm}$.

O objeto simulador foi irradiado em uma unidade de cobaltoterapia Siemens Gammatron II S-80, do Setor de Radioterapia do Hospital das Clínicas da Faculdade de Medicina de Ribeirão Preto da Universidade de São Paulo. Os TLDs foram tratados e lidos no Centro de Instrumentação, Dosimetria e Radioproteção da Universidade de São Paulo (Cidra-USP).

\section{Determinação dos fatores de correção por dependência energética}

Para cada profundidade foram computados os somatórios das energias do espectro multiplicados pela razão entre os respectivos coeficientes de absorção mássicos $\left(\mu_{e n} / \rho\right)$ do LiF e da água ${ }^{(14)}$ em duas parcelas: uma com energias no intervalo de 10 $\mathrm{keV}$ a $670 \mathrm{keV}$ e outra contendo todas as energias presentes no espectro, ou seja, de $10 \mathrm{keV}$ a 1,33 MeV. O cálculo dos fatores de correção foi realizado como apresentado na equação 1 . 


$$
F C_{x}=\frac{\sum_{i=10 \mathrm{keV}}^{i=670 \mathrm{keV}} P_{\mathrm{sec}}\left(E_{i}\right) \cdot E_{i} \cdot\left(\frac{\mu_{e n}\left(E_{i}\right)}{\rho}\right)_{\text {água }}^{L i F}}{\sum_{i=10 \mathrm{keV}}^{i=1,33 \mathrm{MeV}} P\left(E_{i}\right) \cdot E_{i} \cdot\left(\frac{\mu_{e n}\left(E_{i}\right)}{\rho}\right)_{\text {água }}^{L i F}}
$$

Equação 1. Fatores de correção da leitura do LiF-100 por contribuição em dose do espectro secundário de um feixe clínico de Co-60 em profundidade na água.

Na equação $1,\left(\mu_{e n}\left(E_{i}\right) / \rho\right)$ corresponde à razão entre os coeficientes de absorção mássicos $\left(\mu_{e n} / \rho\right)$ do LiF e da água para uma energia $E_{i} ; P\left(E_{i}\right)$ é a densidade de probabilidade de ocorrência de partículas com energia $E_{i}$ na profundidade $x$, relativa ao espectro total; $P_{\text {sec }}\left(E_{i}\right)$ é a densidade de probabilidade de ocorrência de partículas com energia $E_{i}$, renormalizada para o espectro secundário da profundidade $x ; F C_{x}$ é o fator de correção para a leitura do TLD calibrada em dose na profundidade $x$ do objeto simulador.

\section{RESULTADOS}

Foram obtidos espectros em 24 profundidades diferentes do objeto simulador, em intervalos de $2 \mathrm{~mm}$ no primeiro centímetro, a fim de observar o comportamento da região de build-up, e a cada $1 \mathrm{~cm}$ em profundidades após o build-up, varrendo toda a extensão do objeto simulador. $\mathrm{O}$ espectro total simulado na profundidade de 0,5 $\mathrm{cm}$, mostrado na Figura 1, apresenta uma componente espectral de baixa energia com pico em $220 \mathrm{keV}(2,5 \%)$ e com expressão percentual total de $5,5 \%$.

Os espectros secundários simulados para as profundidades de $5,5 \mathrm{~cm}, 10,5 \mathrm{~cm}$,

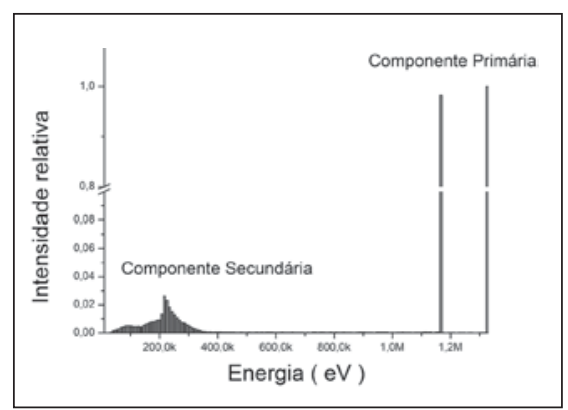

Figura 1. Espectro total de energia na profundidade de máxima dose do Co-60 em água.
$20,5 \mathrm{~cm}$ e $25,5 \mathrm{~cm}$ ao longo do eixo central do objeto simulador são apresentados na Figura 2. As intensidades relativas apresentadas nos espectros simulados têm um máximo na profundidade de $5,5 \mathrm{~cm}$, onde o pico do espectro de baixa energia representa 7\% do espectro total. Após esta profundidade, a contribuição do pico de energia, centrado em $220 \mathrm{keV}$, passa a $5 \% \mathrm{em}$ $10,5 \mathrm{~cm}$, caindo para $1,4 \%$ e $1,2 \%$ em 20,5 $\mathrm{cm}$ e $25,5 \mathrm{~cm}$, respectivamente. Todavia, a $0,95 \%$ de representatividade. contribuição na deposição em dose vai aumentando, pois a expressão relativa de energias maiores que $220 \mathrm{keV}$ cresce.

Para a obtenção $\operatorname{dos} F C_{x}$, foram consideradas as profundidades nas quais havia dados de PDP com ambos os dosímetros (o TLD e a câmara de ionização). A Figura 3A apresenta, graficamente, os valores de PDP medidos com os TLDs, com e sem correção de resposta, juntamente às medidas de PDP feitas com a câmara de ionização e a curva de PDP calculada através da integração dos espectros de energia simulados com o código PENELOPE. As diferenças percentuais entre as leituras de dose absorvida com TLDs, com e sem a aplicação dos $F C_{x}$, e medidas com a câmara de ionização estão apresentadas na Figura 3B.

O gráfico na Figura 3A evidencia o código PENELOPE como adequado e acurado para a simulação de espectros em profundidade na água, convalidando a análise espectral apresentada neste trabalho. Pela

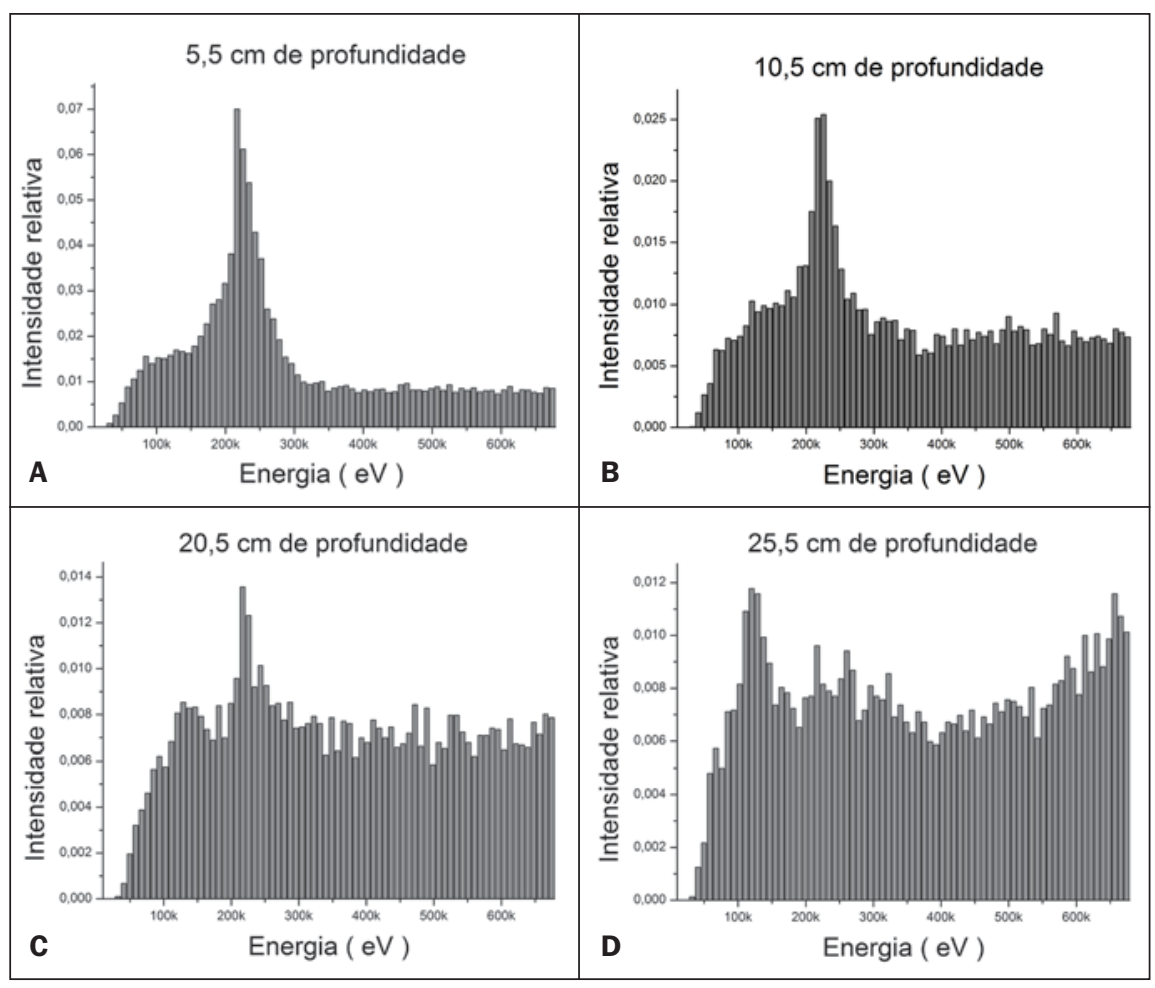

Figura 2. Espectros de energia em profundidade na água, obtidos por simulação Monte Carlo, para um feixe de Co-60 incidindo com um campo quadrado de lado $10 \mathrm{~cm}$ na superfície do objeto simulador. A: Espectro secundário do feixe clínico de Co-60 em 5,5 cm dentro da água: pico de $220 \mathrm{keV} \mathrm{com} \mathrm{7 \%} \mathrm{de}$ representatividade. B: Espectro secundário do feixe clínico de Co-60 em 10,5 cm dentro da água: pico de $220 \mathrm{keV}$ com 2,4\% de representatividade e pico de $600 \mathrm{keV}$ com 0,6\% de representatividade. C: Espectro secundário do feixe clínico de Co-60 em 20,5 cm dentro da água: pico de $220 \mathrm{keV}$ com 1,3\% de representatividade e pico de $600 \mathrm{keV}$ com 0,8\% de representatividade. D: Espectro secundário do feixe clínico de Co-60 em 25,5 cm dentro da água: pico de $220 \mathrm{keV}$ e pico de $600 \mathrm{keV}$, ambos com 


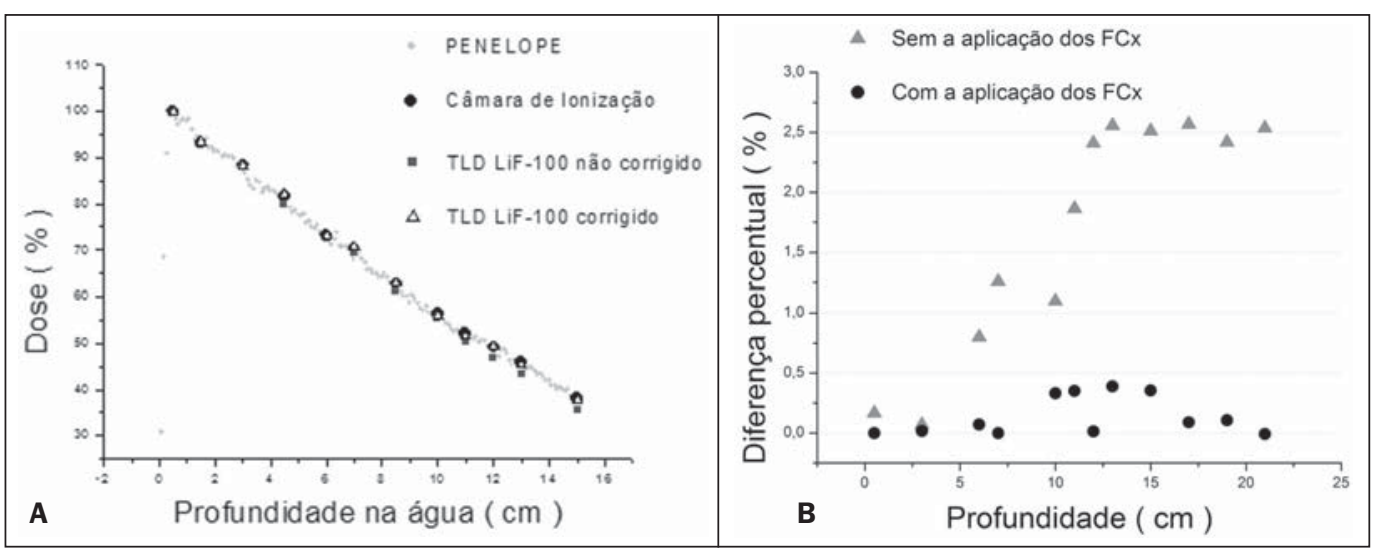

Figura 3. A: Curvas de porcentagem de dose profunda na água para o feixe de Co-60 simulada com o PENELOPE, obtidas com a câmara de ionização e com TLDs de LiF antes e após a aplicação dos fatores de correção. B: Diferenças percentuais entre as medidas da câmara de ionização e dos TLDs antes e após a aplicação dos $F C_{x}$. 0 intervalo de diferenças percentuais é reduzido de até $2,6 \%$ para até $0,3 \%$. análise da Figura 3 é possível visualizar o quanto as leituras com TLDs tornam-se mais imprecisas em maiores profundidades, em razão da presença de radiação de baixas energias, o que comprova a necessidade da aplicação dos $F C_{x}$. Na Figura 3B fica evidente que os $F C_{x}$ aproximam as medidas feitas com TLDs das leituras da câmara de ionização, reduzindo discrepâncias de até 3,2\% para, no máximo, $0,38 \%$. Os dados numéricos dos $F C_{x}$ podem ser vistos na Tabela 1 .

\section{DISCUSSÃO}

A prática clínica da radioterapia com altos índices de qualidade se embasa principalmente numa dosimetria de referência rigorosa, periódica e reprodutível ${ }^{(14,15)}$. Cor- roborando essa afiarmativa, o uso de TLDs na dosimetria em radioterapia se apresenta como uma alternativa complementar à dosimetria de referência, possibilitando testes de controle de qualidade com maior frequência e com relativa simplicidade, sem onerar ou interferir na rotina clínica, demasiadamente $^{(\mathbf{1 6 )}}$

A redução de incertezas e erros associados à dosimetria em radioterapia é um tema foco de inúmeros estudos científicos e clínicos, tendo como maior desafio o fato de que precisão e acurácia dificilmente são encontradas em um único dosímetro ${ }^{(17)}$. $\mathrm{O}$ uso de simulação Monte Carlo, combinado com medidas experimentais com TLDs, para determinar parâmetros dosimétricos diretamente se apresenta como uma alternativa precisa e viável frente à necessidade

Tabela 1 Comparação entre os valores de porcentagem de dose profunda obtidos com os TLDs corrigidos por dependência energética de resposta e os valores medidos com a câmara de ionização.

\begin{tabular}{ccccccc}
\hline \multicolumn{7}{c}{ Porcentagem de dose profunda e fatores de correção para LiF-100 } \\
\hline X (cm) & Cl* $^{*}$ & TLD $^{\dagger}$ & $\Delta \%{ }^{*} \dagger$ & $F C_{x}$ & TLD $_{\text {corrigido }}^{\ddagger}$ & $\Delta^{*}{ }^{*} \neq$ \\
\hline 0,5 & 100,0 & 100,00 & 0,166 & 1,0017 & 100,00 & 0,001 \\
3 & 88,4 & 88,33 & 0,069 & 1,0096 & 88,38 & 0,018 \\
6 & 73,2 & 72,40 & 0,800 & 1,0101 & 73,13 & 0,073 \\
7 & 70,6 & 69,34 & 1,257 & 1,0181 & 70,60 & 0,001 \\
10 & 56,4 & 55,30 & 1,100 & 1,0199 & 56,07 & 0,332 \\
11 & 52,0 & 50,17 & 1,864 & 1,0302 & 51,68 & 0,351 \\
12 & 49,2 & 46,79 & 2,410 & 1,0412 & 49,19 & 0,014 \\
13 & 45,8 & 43,24 & 2,557 & 1,0501 & 45,41 & 0,389 \\
15 & 38,1 & 35,59 & 2,512 & 1,0606 & 37,74 & 0,356 \\
17 & 32,5 & 29,93 & 2,568 & 1,0828 & 32,41 & 0,089 \\
19 & 29,3 & 26,88 & 2,418 & 1,0860 & 29,19 & 0,107 \\
21 & 27,0 & 24,46 & 2,536 & 1,1040 & 27,01 & 0,008 \\
\hline
\end{tabular}

${ }^{*} \mathrm{Cl}$, medidas experimentais com a câmara de ionização; ${ }^{\dagger} \mathrm{TLD}$, medidas experimentais com TLDs LiF-100; $\Delta \%$, diferença percentual; $F C_{x}$, fatores de correção calculados com PENELOPE; ${ }^{\ddagger} \mathrm{TLD}_{\text {corrigido }}$, medidas de dose absorvida no TLD corrigidas pelo fator de correção $F C_{x}$.

de redução de incertezas e erros em radioterapia. Com a consideração dos fatores de correção apresentados neste trabalho é possível utilizar TLDs de LiF para medidas de PDP em água, com um intervalo de erro relativo à câmara de ionização coerente com os índices recomendados pela literatura de referência em controle de qualidade de feixes radioterápicos.

A análise espectral baseada nos espectros totais e secundários de fótons em profundidade na água, simulados com o código PENELOPE ${ }^{(\mathbf{1 8 , 1 9})}$, permite o cálculo de fatores de correção que podem ser aplicados de maneira relativamente simples. Os espectros de fótons em profundidade na água permitem observar a existência de baixas energias na profundidade de máxima dose, distribuindo-se entre 30 e $350 \mathrm{keV}$, região na qual a resposta do $\mathrm{LiF}$ tem comportamento não linear e alterna-se entre uma resposta relativa decrescente em energias de 30 a $100 \mathrm{keV}$ e suavemente crescente em energias no intervalo de 100 a 300 $\mathrm{keV}$, aproximadamente.

Em todos os espectros simulados até $15,5 \mathrm{~cm}$ dentro do objeto simulador o pico de baixa energia se mantém em $220 \mathrm{keV}$ e a expressão das parcelas com energias de 30 a $300 \mathrm{keV}$ é predominante, o que deixa de ocorrer em profundidades abaixo da metade do objeto simulador, nas quais as probabilidades de ocorrência de partículas com baixa energia passam a se distribuir de forma equânime em um intervalo maior, de 30 a $670 \mathrm{keV}$.

À medida que a profundidade aumenta, os espectros totais apresentam um maior número de partículas com energias mais baixas, o que desloca a energia média do 
feixe simulado que atinge aquela profundidade para um valor menor do que a energia média do feixe de Co-60 incidente, que seria 1,25 MeV, comumente considerada para os cálculos de energia absorvida em profundidade na água.

É possível observar que a área dos espectros secundários com energias entre 10 $\mathrm{keV}$ e $670 \mathrm{keV}$ se mantém aproximadamente a mesma após a profundidade de build-up, deslocando, portanto, a energia efetiva do espectro secundário que aumenta a dose em cada detector. Este aumento não é devidamente observado em medidas experimentais realizadas com TLDs de LiF, em razão da sua dependência energética de resposta exatamente nesta faixa de energia, o que pode ser observado nas curvas de PDP obtidas com estes dosímetros (ver Figura 3A).

Apesar do decréscimo da energia média com a profundidade ser facilmente visualizado a partir da observação dos espectros simulados, um estudo mais preciso da dose absorvida em uma dada profundidade deve considerar o $\left(\mu_{e n} / \rho\right)$ correspondente a cada conjunto discreto de partículas com uma dada energia, que são, por sua vez, absorvidos de forma diferente pela água e pelo $\mathrm{LiF}$, gerando doses depositadas diferentes em cada um desses meios. É exatamente observando as parcelas de baixa energia e sua significância em cada profundidade que é possível relacionar as diferenças de absorção entre o meio de interesse, a água, e o material do dosímetro, $\mathrm{LiF}$, aplicando os $F C_{x}$ determinados neste trabalho.

Quando comparadas, as curvas de PDP obtidas com uma câmara de ionização e com TLDs apresentam uma diferença percentual que aumenta com a profundidade, de $0,2 \%$ a $3,2 \%$, se mantendo aproximadamente constante, em torno de $2,5 \%$, para profundidades maiores que $10 \mathrm{~cm}$. Observando ainda que a probabilidade relativa das partículas com baixas energias aumenta com a profundidade no objeto simulador (análise da Figura 2), e considerando-se que o número total de partículas simuladas em cada interação se mantém o mesmo, é fácil notar que o $F C_{x}$ deve crescer com a profundidade, como mostrado na Tabela 1.

\section{CONCLUSÃO}

A análise espectral baseada na simulação Monte Carlo de um feixe clínico de Co60 possibilita o cálculo de fatores de correção satisfatórios para as leituras de dose absorvida em TLDs de LiF. A aplicação dos fatores de correção $F C_{x}$ nas leituras de TLDs contribui para o aumento da precisão na determinação de parâmetros dosimétricos intimamente associados ao controle de qualidade de feixes clínicos em radioterapia.

Os resultados apresentados neste trabalho podem ser estendidos para diversos protocolos clínicos, a fim de tornar o uso de TLDs mais acurado e rotineiro.

\section{Agradecimentos}

Ao Setor de Radioterapia do Hospital das Clínicas da Faculdade de Medicina de Ribeirão Preto (HCFMRP-USP) e ao Centro de Instrumentação Dosimetria e Radioproteção da Universidade de São Paulo (Cidra-USP).

\section{REFERÊNCIAS}

1. Kawa-Iwanicka A, Lobodziec W, Iwanicki T, et al. Dose uniformity in the total body irradiation technique using $15 \mathrm{MV}$ photon beam. Physica Medica. 2004;20(Suppl 1):144-6.

2. International Atomic Energy Agency. Absorbed dose determination in external beam radiotherapy. An International Code of Practice for Dosimetry Based on Standards of Absorbed Dose to Water. Technical Reports Series No. 398. Vienna: International Atomic Energy Agency; 2000.

3. International Commission on Radiation Units and Measurements. Measurement of absorbed dose in a phantom irradiated by a single beam of $\mathrm{X}$ or gamma rays. ICRU Report 23. Bethesda: International Commission on Radiation Units and Measurements; 1973.

4. Vrtar M. A dosimetric method of total body irradiation. Cell Mol Biol Lett. 2002;7:337-40.

5. Zabatis Ch, Koligliatis T, Xenofos S, et al. Dosimetry in translation total body irradiation technique: a computer treatment planning approach and an experimental study concerning lung sparing. J Buon. 2008;13:253-62.

6. Bloemen-van Gurp EJ, Mijnheer BJ, Verschueren TA, et al. Total body irradiation, toward optimal individual delivery: dose evaluation with metal oxide field effect transistors, thermoluminescence detectors, and a treatment planning system. Int J Radiat Oncol Biol Phys. 2007;69:1297-304.

7. Kron T. Thermoluminescence dosimetry and its applications in medicine-Part 1: Physics, materials and equipament. Australas Phys Eng Sci Med. 1994;17:175-99.

8. Giordani AJ, Segreto HRC, Segreto RA, et al. Verificação das doses de radiação absorvidas durante a técnica de irradiação de corpo inteiro nos transplantes de medula óssea, por meio de dosímetros termoluminescentes. Radiol Bras. 2004;37:343-9.

9. Reyes FE. Procedimento de calibração de dosímetros termoluminescentes em dose absorvida na água para fontes de irídio- ${ }^{192} \mathrm{I}$ de alta taxa de dose [dissertação de mestrado]. Rio de Janeiro: Instituto de Radioproteção e Dosimetria; 2004.

10. Almeida CE, Affonseca M, Calcina CSG, et al. Rastreabilidade das referências metrológicas em dose absorvida na água do Programa de Qualidade em Dosimetria. Radiol Bras. 2005;38:205-8.

11. Garnica-Garza HM. Monte Carlo-derived TLD cross-calibration factors for treatment verification and measurement of skin dose in accelerated partial breast irradiation. Phys Med Biol. 2009;54: 1621-31.

12. Panettieri V, Sempau J, Andreo P. Chamber-quality factors in ${ }^{60} \mathrm{Co}$ for three plane-parallel chambers for the dosimetry of electrons, protons and heavier charged particles: PENELOPE Monte Carlo simulations. Phys Med Biol. 2008;53: 5917-26.

13. International Commission on Radiation Units and Measurements. Tissue substitutes in radiation dosimetry and measurement. ICRU Report 44. Bethesda: International Commission on Radiation Units and Measurements; 1989.

14. Morávek Z, Rickhey M, Hartmann M, et al. Uncertainty reduction in intensity modulated proton therapy by inverse Monte Carlo treatment planning. Phys Med Biol. 2009;54:4803-19.

15. Guckenberger M, Krieger T, Richter A, et al. Potential of image-guidance, gating and real-time tracking to improve accuracy in pulmonary stereotactic body radiotherapy. Radiother Oncol. 2009;91:288-95

16. McGarry CK, Cosgrove VP, Fleming VA, et al. An analysis of geometric uncertainty calculations for prostate radiotherapy in clinical practice. $\mathrm{Br} \mathrm{J}$ Radiol. 2009;82:140-7.

17. Budrukkar A, Dutta D, Sharma D, et al. Comparison of geometric uncertainties using electronic portal imaging device in focal three-dimensional conformal radiation therapy using different head supports. J Cancer Res Ther. 2008;4:70-6.

18. Salvat F, Fernández-Varea JM, Acosta E, et al. PENELOPE - a code system for Monte Carlo simulation of electron and photon transport. Paris: Nuclear Energy Agency; 2008.

19. Salvat F, Fernández-Varea JM. Overview of physical interaction models for photon and electron transport used in Monte Carlo codes. Metrologia. 2009; 46:S112-38. 